\title{
Philosophiques
}

\section{Théorie du raisonnement et perspective de la première personne}

\section{Josée Brunet}

Volume 37, numéro 2, automne 2010

URI : https://id.erudit.org/iderudit/045190ar

DOI : https://doi.org/10.7202/045190ar

Aller au sommaire du numéro

Éditeur(s)

Société de philosophie du Québec

ISSN

0316-2923 (imprimé)

1492-1391 (numérique)

Découvrir la revue

Citer cet article

Brunet, J. (2010). Théorie du raisonnement et perspective de la première personne. Philosophiques, 37(2), 411-437. https://doi.org/10.7202/045190ar
Résumé de l'article

Partant de l'hypothèse qu'une théorie adéquate du raisonnement devrait être en mesure de répondre à la question de savoir comment les aspects processuel (psychologique) et inférentiel (logique) d'un raisonnement sont liés entre eux, l'objectif de cet article est de déterminer comment Gilbert Harman conçoit cet aspect processuel, et si sa théorie permet d'expliquer de manière adéquate la relation entre ces deux aspects. J'examine d'abord quelle fonction il reconnaît aux notions d'implication et d'inconsistance, et montre ensuite que si le processus dans lequel s'engage celui qui raisonne peut être conçu à la manière d'Harman, c'est-à-dire à la $3^{\mathrm{e}}$ personne, il doit aussi être conçu du point de vue de l'agent qui raisonne, c'est-à-dire à la $1^{\text {re }}$ personne. Après avoir discuté des limites inhérentes au fait de concevoir l'aspect processuel du raisonnement d'un point de vue essentiellement externe, à la $3^{\mathrm{e}}$ personne, j'identifie quelques conséquences que fait apparaître la perspective de la $1^{\text {re }}$ personne, dans le but de montrer pourquoi une théorie adéquate du raisonnement ne peut en faire l'économie. 


\title{
Théorie du raisonnement et perspective de la première personne
}

\author{
JOSÉE BRUNET \\ Institut de technologie agroalimentaire - Campus de Saint-Hyacinthe
}

\begin{abstract}
RÉSUMÉ. - Partant de l'hypothèse qu'une théorie adéquate du raisonnement devrait être en mesure de répondre à la question de savoir comment les aspects processuel (psychologique) et inférentiel (logique) d'un raisonnement sont liés entre eux, l'objectif de cet article est de déterminer comment Gilbert Harman conçoit cet aspect processuel, et si sa théorie permet d'expliquer de manière adéquate la relation entre ces deux aspects. J'examine d'abord quelle fonction il reconnaît aux notions d'implication et d'inconsistance, et montre ensuite que si le processus dans lequel s'engage celui qui raisonne peut être conçu à la manière d'Harman, c'est-à-dire à la $3^{\mathrm{e}}$ personne, il doit aussi être conçu du point de vue de l'agent qui raisonne, c'est-à-dire à la $1^{\text {re }}$ personne. Après avoir discuté des limites inhérentes au fait de concevoir l'aspect processuel du raisonnement d'un point de vue essentiellement externe, à la $3^{\mathrm{e}}$ personne, j'identifie quelques conséquences que fait apparaître la perspective de la $1^{\text {re }}$ personne, dans le but de montrer pourquoi une théorie adéquate du raisonnement ne peut en faire l'économie.
\end{abstract}

\begin{abstract}
Assuming that an adequate account of reasoning should be able to explain how the processual (psychological) aspect of a piece of reasoning relates to its inferential (logical) aspect, this article seeks to determine how Gilbert Harman's conceives of this processual aspect, and whether his theory allows for a correct explanation of its relation to the inferential aspect. I consider first which role he attributes to the notions of implication and consistency, and then argue that even though the process in which a reasoning subject engages can be thought of in the way suggested by Harman, i.e., from the $3^{\text {rd }}$ person perspective, it must also be conceived from the $1^{\text {st }}$ person perspective, from the point of view of the reasoning subject. I discuss some of the unavoidable limitations of conceiving the processual aspect of reasoning from such an essentially external point of view, and draw attention to some of the advantages of adopting the $7^{\text {st }}$ person perspective, with the aim of showing why no adequate theory of reasoning could completely dispense with it.
\end{abstract}

\section{Propositions de départ'}

Raisonner a longtemps été conçu comme une enquête "argumentative», au sens où les raisonnements ou "inférences" ont souvent été compris et analysés essentiellement en termes propositionnels (en tant que relations entre des propositions). La tâche de la logique fut, par conséquent, longtemps comprise comme celle de repérer les règles (logiques) qui déterminent la validité de l'inférence (argument), et par conséquent conçue comme responsable

1. Je tiens à remercier Christine Tappolet, Serge Robert et tout particulièrement Daniel Laurier de leurs commentaires et suggestions. 
de définir en quoi consiste le fait de raisonner correctement (d'élaborer une théorie normative du raisonnement). Traditionnellement, cela conduisit à assimiler le raisonnement d'un agent à un syllogisme (ou un argument), ce qui a finalement obscurci substantiellement l'idée qu'un raisonnement est d'abord et avant tout un processus mental. Cette manière de concevoir le raisonnement a conduit à différentes erreurs, notamment celle d'assimiler les termes «raisonnement correct» et "argument valide" (à assimiler les principes du raisonnement à ceux de la logique). Pourtant, un raisonnement n'est pas un argument, et raisonner ne consiste pas à suivre les règles ou principes de la logique. Le raisonnement se distingue précisément de l'argument par son caractère psychologique, c'est-à-dire en vertu du fait que l'agent qui raisonne s'engage dans un processus qui met en relation ses propres états mentaux.

Cette idée de restreindre le rôle de la logique dans l'étude du raisonnement et de concevoir le raisonnement comme un processus (plutôt que comme un argument) n'est pas nouvelle. Elle a notamment été défendue par Gilbert Harman (1976, 1986, 1999, 2007ms), pour qui raisonner est un processus dans lequel nous nous engageons pour réviser nos croyances, nos intentions et nos plans, et la théorie du raisonnement qu'il propose dans Harman (1986) consiste précisément à identifier quels sont ces principes que nous suivons/devons suivre lorsque nous raisonnons. Néanmoins, dire qu'un raisonnement n'est pas un argument ne revient pas à dire qu'il est impossible de lui associer un argument qui met en relation les contenus propositionnels de certains des états mentaux impliqués dans le raisonnement proprement dit d'un agent. Négliger cette possibilité reviendrait (à l'inverse d'ignorer l'aspect processuel du raisonnement sur lequel insiste Harman) à ignorer cet autre aspect, cette fois inférentiel, qui est aussi spécifique au fait de raisonner. J'aimerais ainsi montrer dans cet article que (i) une théorie adéquate du raisonnement ne peut se confiner à l'aspect processuel du raisonnement dans la mesure où s'en tenir à ce seul aspect conduit à adopter un point de vue externe à l'agent (point de vue de la $3^{\text {e }}$ personne); (ii) certaines des limites de la théorie développée par Harman reposent sur cette difficulté; (iii) par conséquent, bien qu'une théorie adéquate du raisonnement ne puisse faire l'économie du point de vue de la $3^{\mathrm{e}}$ personne, elle doit réussir à rendre compte de manière adéquate de la perspective de l'agent qui raisonne (le point de vue de la $1^{\text {re }}$ personne), qui, elle, renvoie à l'aspect inférentiel de son raisonnement.

L'hypothèse sur laquelle je m'appuie dans les pages qui suivent est donc qu'un raisonnement possède un double aspect: d'une part, un aspect processuel, par le fait qu'il met en relation les états mentaux de l'agent qui raisonne; d'autre part, un aspect inférentiel, puisque l'agent qui raisonne met en relation les contenus propositionnels de certains de ses états mentaux. La thèse qui sera mise de l'avant est la suivante: associer un argument au raisonnement proprement dit d'un agent consiste en fait à référer à l'aspect inférentiel du raisonnement. Cet argument n'est pas le raisonnement de 
l'agent: il n'est tout au plus qu'une Représentation du Contenu de certains des États mentaux impliqués dans le raisonnement proprement dit de cet agent ${ }^{2}$. Je suggère de nommer cet argument, "ReCEM", et utiliserai cet acronyme pour y référer. Les lettres qui le composent sont les abréviations des termes « représentation» $(\operatorname{Re})$, «contenu » $(\mathrm{C})$, « états $(\mathrm{E})$, et «mentaux» $(\mathrm{M})$. Puisqu'il s'agit d'un argument, il est, à l'inverse du raisonnement proprement dit, composé de prémisses et d'une conclusion, et soumis aux critères de validité logique, c'est-à-dire aux règles logiques qui s'appliquent aux arguments. J'aimerais montrer que la relation que l'on peut établir entre le raisonnement proprement dit d'un agent et l'argument ReCEM qu'on peut lui associer illustre quelque chose de particulier: d'une part, bien que le point de vue (subjectif) d'un agent puisse se manifester dans sa manière «d'appréhender» un argument, étrangement, rien dans l'argument luimême ne permet de traduire ce point de vue (subjectif). En d'autres termes, aucun argument ReCEM ne pourrait réussir à capturer le point de vue (subjectif) de l'agent, notamment le fait qu'il représente ou non le contenu de certains états mentaux impliqués dans son raisonnement proprement dit, puisqu'il ne s'agit que d'une relation entre des propositions. D'autre part, et tout aussi étrangement, il semble que seul cet argument serait en mesure de rendre compte de l'aspect inférentiel du raisonnement auquel il est associé, même s'il ne consiste qu'en une représentation du contenu des états mentaux impliqués dans le raisonnement proprement dit de l'agent. C'est peutêtre ce qui a conduit certains à confondre ou à assimiler le raisonnement à un argument, et à supposer que raisonner consiste à suivre des principes logiques. À tout le moins, cela semble avoir conféré un caractère énigmatique à la question de savoir comment concevoir la relation entre les aspects processuel et inférentiel du raisonnement.

Si cela est juste, il semble que l'une des tâches qu'une théorie du raisonnement a à relever est de rendre compte de manière adéquate de cette relation. En d'autres termes, une théorie adéquate du raisonnement devrait être en mesure de répondre à la question de savoir comment les aspects processuel et inférentiel d'un raisonnement sont liés entre eux. Cette question n'est pas nouvelle, et certains auteurs ont tenté d'y répondre: n'est-ce pas précisément une solution à ce genre de question que cherche Anscombe

2. Illustrons le caractère évident de cette distinction à l'aide de l'exemple suivant. Supposons que vous demandiez à Anna qui croit que tous et seulement les oxoniens ont publié un livre chez Oxford et croit que Sam est un oxonien, si Sam a publié chez Oxford, et qu'elle vous réponde "oui, puisqu'il est oxonien et que tous et seulement les oxoniens ont publié chez Oxford ». De manière évidente, elle ne peut répondre "oui » (de manière rationnelle) que dans la mesure où «tous et seulement les oxoniens ont publié chez Oxford» et "Sam est un oxonien" sont les contenus de ses propres états mentaux. Il est alors possible de faire correspondre à son raisonnement un argument, mais dans la mesure où un argument est une mise en relation de propositions, et un raisonnement, une mise en relation d'états mentaux, cet argument n'est pas son raisonnement: il permet tout au plus de référer à l'aspect inférentiel de son raisonnement. 
(1957) lorsqu'elle affirme, à la suite d'Aristote, que l'action est la conclusion du raisonnement pratique ou syllogisme pratique et tente de traduire l'aspect processuel du raisonnement en termes inférentiels ${ }^{3}$ ?

D'autre part, du moment où l'on reconnaît qu'un raisonnement n'est pas un argument mais bien un processus psychologique, une autre question importante à laquelle une théorie du raisonnement devrait être en mesure de répondre concerne ce qui distingue ce type de processus mental des autres processus mentaux d'un agent, c'est-à-dire quelles en sont les caractéristiques spécifiques (qu'est-ce qui distingue le fait de raisonner de celui d'imaginer, par exemple, ou d'autres processus mentaux), et qu'est-ce qui détermine si le processus est correct ou non (qu'est-ce qui fait que quelqu'un raisonne correctement ou non, qu'il a respecté ou non les normes du raisonnement correct). Si l'on s'en tient à l'idée traditionnelle que raisonner donne à l'être humain la possibilité de déterminer ce qu'il est rationnel de croire/ faire, on pourrait s'attendre à ce que, lorsqu'un agent raisonne correctement, les attitudes acquises ou abandonnées au terme de ce processus sont rationnelles à la lumière de l'ensemble de ses états mentaux. Mais il n'est pas sûr, à mon avis, que cette deuxième question puisse être résolue indépendamment de la première, c'est-à-dire qu'il soit possible de caractériser adéquatement l'aspect processuel du raisonnement sans prendre en considération la manière dont il est lié à cet autre aspect qui, lui, est inférentiel.

La conjecture à l'origine de cet article est ainsi la suivante: la possibilité d'expliquer de manière adéquate la relation entre les aspects processuel et inférentiel du raisonnement, et donc de disposer d'une théorie adéquate du raisonnement, reposerait (au moins en partie) sur une compréhension adéquate de ce qui distingue et lie le raisonnement proprement dit d'un agent, et l'argument ReCEM qu'on peut lui associer. Ce qui renvoie à la première question identifiée, c'est-à-dire celle de savoir comment les aspects processuel et inférentiel sont liés entre eux. J'ai suggéré qu'il était peu probable que l'on puisse caractériser le raisonnement compris comme processus sans que cette question soit prise en considération. Pourtant, la théorie du raisonnement développée par Harman semble (du moins à première vue) vouloir ignorer cette question et se limiter à répondre à la deuxième, dont l'enjeu est de définir en quoi consiste ce processus.

L'objectif de cet article est donc principalement de déterminer comment Harman conçoit cet aspect processuel, et d'examiner s'il n'est pas

3. Je discute de cette question dans Brunet, 2003, p. 479-500. Je montre que la proposition d'Anscombe qui consiste à s'opposer à ce que l'argument pratique soit caractérisé en termes de validité logique est partiellement juste, mais n'est juste que dans la mesure où elle s'applique à toute forme de raisonnement que l'on voudrait traduire par un argument. J'explique pourquoi, en voulant éviter de nier l'aspect processuel du raisonnement, Anscombe rend compte de manière inadéquate de la relation entre les aspects processuel et inférentiel du raisonnement lorsqu'elle suggère (à la suite d'Aristote) que l'action — un événement plutôt qu'une proposition - est la conclusion du syllogisme pratique. 
conduit indirectement à prendre en considération la relation entre les aspects processuel et inférentiel du raisonnement. Le texte est divisé de la manière suivante: j'examine d'abord comment Harman choisit de distinguer les notions de raisonnement et d'argument, et tente ensuite de déterminer s'il réserve une fonction à l'argument ReCEM dans sa théorie du raisonnement, notamment en exposant quelle fonction il reconnait aux notions d'implication et d'inconsistance dans le processus de révision. Je montre ensuite que le processus dans lequel s'engage celui qui raisonne peut être conçu à la manière d'Harman (à la $3^{e}$ personne), mais aussi du point de vue de l'agent qui raisonne (à la $1^{\text {re }}$ personne), et discute des limites inhérentes au fait de concevoir l'aspect processuel du raisonnement d'un point de vue essentiellement externe (à la $3^{\mathrm{e}}$ personne), notamment en montrant comment les principes d'implication reconnue et d'inconsistance reconnue que Harman admet lui-même comme principes de révision illustrent l'impossibilité de faire abstraction de la perspective de la $1^{\text {re }}$ personne. En guise de conclusion, j'identifie quelques conséquences que met en lumière la perspective de la $1^{\text {re }}$ personne (qui révèlent les limites de l'approche d'Harman) et montre pourquoi une théorie adéquate du raisonnement ne peut en faire l'économie.

\section{La distinction raisonnement/argument selon Harman}

La nécessité de limiter le rôle de la logique dans l'étude du raisonnement est une des thèses centrales sur laquelle Harman fonde sa théorie du raisonnement, et la nécessité de distinguer "raisonnement» et «argument» en découle directement. Examinons en premier lieu ce qui nous oblige, selon lui, à faire cette distinction. Il affirme (1999):

Issues about inference and reasoning need to be distinguished from issues about implication and consistency. Inference and reasoning are psychological processes leading to possible changes in belief (theoretical reasoning) or possible changes in plans and intentions (practical reasoning). Implication is most directly a relation among propositions. Certain propositions imply another proposition when and only when, if the former propositions are true, so too is the latter proposition ${ }^{4}$.

Harman associe le terme "inférence» à l'activité de raisonner et insiste sur la nécessité de distinguer les questions qui portent sur l'inférence et le raisonnement de celles qui portent sur l'implication et la consistance. Harman vise juste: un raisonnement n'est pas un argument, dans la mesure où un raisonnement est un processus psychologique qui met en relation des états mentaux et qu'un argument est une relation entre des propositions (prémisses et conclusion), d'où l'importance de distinguer, comme je l'ai suggéré plus haut, le raisonnement proprement dit de l'agent et l'argument ReCEM qu'on peut lui associer. Notons qu'Harman n'utilise pas ici le

4. Harman, 1999, p. 18. 
terme "argument» et préfère parler de "issues about implication and consistency $»^{5}$.

Dans «Practical Reasoning» (1999), Harman précise pourquoi il est nécessaire de distinguer le raisonnement de l'argument, c'est-à-dire pourquoi le raisonnement doit être conçu essentiellement comme un processus, et pourquoi l'argument n'est pas le raisonnement proprement dit d'un agent (an instance of reasoning) ${ }^{6}$. Selon lui, un raisonnement n'a pas de prémisses ni de conclusion, à moins d'admettre que toutes les croyances et les intentions initiales d'un agent fassent partie des prémisses $d u$ raisonnement et que la conclusion corresponde au nouvel ensemble d'états mentaux de l'agent. Il ajoute que même si on admettait cette idée, on ne réussirait pas à rendre compte de l'activité de raisonner comme telle, puisque celle-ci nous oblige

5. Une remarque terminologique s'impose toutefois quant à l'usage qu'il fait du terme «inférence». Harman l'associe à celui de «raisonnement». J'ai suggéré de ranger ce terme plutôt du côté de l'argument, et choisi de faire correspondre l'aspect inférentiel du raisonnement à la relation entre les contenus propositionnels de certains des états mentaux impliqués dans le raisonnement proprement dit de l'agent. Certains pourraient considérer l'usage qu'Harman fait du terme « inférence " plus intuitif, dans la mesure où inférer peut très bien être conçu comme une activité, un processus psychologique, et si tel est le cas une "inférence» ressemblerait plus à un raisonnement qu'à un argument. Intuitivement, on pourrait par conséquent donner raison à Harman et considérer que ma suggestion est contre-intuitive. Néanmoins, ce qui la motive vient de la confusion entourant l'usage du terme «inférence " lorsque vient le temps de comprendre la relation qui existe entre un raisonnement et un argument ReCEM. Dans la mesure où les raisonnements (ou inférences selon Harman) sont des processus psychologiques (qui mettent en relation des états mentaux, lesquels possèdent des contenus propositionnels) et que les arguments sont des relations entre des propositions (qui peuvent être des représentations de contenus d'états mentaux impliqués dans des raisonnements), cela explique, à mon avis, qu'un double usage du terme «inférence » ait fait surface et que les inférences aient été conçues tantôt comme des raisonnements, tantôt comme des arguments (que les règles d'inférence aient été conçues tantôt comme des règles du raisonnement, et tantôt comme des règles logiques). La terminologie proposée par Harman serait admissible si elle permettait de lever clairement cette confusion et de faire la lumière entre les aspects processuel et inférentiel du raisonnement. Je suis d'avis, comme l'indique Harman, que les questions concernant le raisonnement doivent être distinguées de celles concernant l'implication (et la consistance). Je crois néanmoins qu'il est nécessaire de distinguer (du moins pour l'instant) les termes «inférence» et "raisonnement", l'essentiel de ma suggestion étant en fait de limiter (voire d'exclure) l'usage du terme "inférence" dans l'étude du raisonnement pour éviter de retomber dans ce genre de confusion.

6. Il affirme: «Reasoning is here to be taken to be distinguished from proof or argument in a logician's sense. Reasoning is a process of modifying antecedent beliefs and intentions, perhaps by adding some new ones, perhaps by deleting some of the original ones - normally by adding some and deleting others. An argument or proof is sometimes relevant to reasoning in this sense but is never an instance of it. An argument or proof is more like an explanation than an instance of reasoning. It has premises, intermediate steps, and a conclusion. Reasoning has no premises and no conclusion, unless we are to say that the "premises" comprise all of the antecedent beliefs and intentions and that the "conclusion" is the resulting set. But that way of speaking might be misleading, since reasoning often leads to abandoning some "premises" " (Harman, 1999, p. 46). 
souvent à abandonner (au cours du processus) certaines prémisses. D'où la nécessité de distinguer l'activité de raisonner de l'argument lui-même: un argument ou une preuve est parfois pertinent au raisonnement mais n'est jamais un exemple de raisonnement. Pour qu'un argument puisse «traduire » un raisonnement, il faudrait, selon Harman, que les prémisses de cet argument incluent l'ensemble des états mentaux de l'agent au sein des prémisses à $t$, et exposer en conclusion le nouvel ensemble d'états mentaux de l'agent à $t+1$, ceci n'excluant pas la possibilité que durant le passage de $t$ à $t+1$, certaines prémisses soient abandonnées (de manière à tenir compte du caractère processuel).

Examinons si cette manière de distinguer le raisonnement de l'argument implique, si l'on suit l'analyse de Harman, qu'un argument ne peut pas représenter le contenu de certains des états mentaux impliqués dans un raisonnement proprement dit. À première vue, rien n'indique que Harman soit tenu de s'engager dans cette voie ${ }^{7}$. Sa suggestion vise essentiellement à montrer qu'un raisonnement proprement dit ne peut pas avoir la forme d'un argument (elle ne porte pas sur le fait que certains arguments puissent mettre en relation des contenus propositionnels d'états mentaux d'agents, ni sur le fait que ces arguments pourraient avoir des relations avec nos raisonnements proprement $\left.\operatorname{dits}^{8}\right)$. La question que j'aimerais ainsi examiner est celle de savoir si Harman entend réserver un rôle à l'argument ReCEM dans son étude du raisonnement une fois qu'il a admis que le raisonnement proprement dit d'un agent ne peut être assimilé à un argument. Il précise (du moins en partie) comment il conçoit ce rôle dans ce qui suit:

But logic is not directly a theory of reasoning. There is deductive logic but no such thing as deductive reasoning; given a deductive argument, one can always abandon a premise rather than accept the conclusion. There is inductive reasoning (perhaps better called theoretical reasoning) but no such thing as

7. La conception qu'Harman se fait de l'argument est néanmoins distincte de celle que j'ai proposée jusqu'ici: il semble présupposer que si un raisonnement pouvait être traduit par un argument, les prémisses de l'argument coïncideraient alors avec les états mentaux de l'agent. Ce qui (à mon avis) est erroné. J'ai proposé qu'un argument ne met pas en relation les états mentaux de l'agent mais bien les contenus propositionnels: il s'agit d'une relation entre des propositions. Je discuterai plus loin des raisons qui conduisent Harman à cette confusion. Tenons-nous en pour l'instant à l'idée qu'un raisonnement ne peut pas être, selon Harman, un argument, ce qui s'accorde avec ma position.

8 Dans «Notes on Practical Reasoning» (article non publié, juin 2007), Harman précise: "The word "reasoning" can also be used to refer to a product of the process of reasoning. Something written down, for example. I don't want to confuse the process with the product, so I here use "reasoning" just for the process. The product of reasoning might be a linear sequence of sentences or propositions, perhaps in the form of a linear argument. It does not follow that the process of reasoning is linear. It almost certainly involves highly parallel processes of constraint satisfaction", p. 1 . 
inductive logic. Again, there is practical reasoning and no such thing as practical syllogism? .

Ces thèses sont centrales à la théorie du raisonnement de $\operatorname{Harman}^{10}$. Je les résumerai ainsi:

(H1) Nous ne raisonnons pas déductivement mais pouvons raisonner inductivement;

(H2) Si nous ne raisonnons pas déductivement, c'est parce qu'un agent a toujours la possibilité d'abandonner la prémisse d'un argument déductif plutôt que d'accepter la conclusion ${ }^{11}$;

(H3) Puisque nous ne raisonnons pas déductivement, les arguments déductifs ne représentent pas d'intérêt particulier dans l'étude du raisonnement ${ }^{12}$;

(H4) Bien que nous puissions raisonner de manière inductive, il n'y a pas de logique inductive (ni d'argument inductif ${ }^{13}$ ).

Ainsi, du fait que, pour lui, les arguments déductifs n'ont pas de rôle proprement dit à jouer dans une théorie du raisonnement (puisque nous ne raisonnons pas déductivement), et qu'il n'existe ni logique inductive ni argument inductif, Harman conclut qu'une théorie du raisonnement peut être élaborée de manière indépendante de la notion d'argument ( $\mathrm{du}$ moins

9. Harman, 1999, p. 46.

10. Ces thèses sont développées dans les deux premiers chapitres d'Harman, 1986, p. 1-20, et résumées dans Harman, 1999, p. 27-32.

11. Cette thèse mérite d'être questionnée. Comment pourrions-nous vouloir abandonner cette prémisse lorsque nous acceptons la conclusion, si ce n'est que parce que nous raisonnons (au moins en partie) déductivement, que nous suivons (au moins en partie) les règles de l'implication et désirons être consistant?

12. «Indeed, if we clearly distinguish reasoning from argument, we cannot suppose that the existence of deductive arguments shows that there is such a thing as deductive reasoning, that is, deductively reasoned change in view. As I have already observed, rules of deduction are rules of deductive arguments, not rules of inference or reasoning» (Harman, 1986, p. 5).

13. "Now, why should we think there are inductive arguments and an inductive logic? It is clear enough that there is something that might be called inductive reasoning, that is, inductively reasoned change in view. But if we clearly distinguish reasoned change in view from argument, we cannot suppose that the existence of inductive reasoning by itself shows there is such a thing as inductive argument, nor can we suppose that it shows there is an inductive logic» (Harman, 1986, p. 5).

Selon Harman, le fait que nous puissions raisonner de manière inductive n'impliquerait pas l'existence d'arguments inductifs ou d'une logique inductive. Cette citation doit être comprise à la lumière de celle faite précédemment: il n'y a pas (selon lui) de logique inductive, ni d'argument pratique (ni de syllogisme pratique). Je crois néanmoins qu'à première vue, rien ne nous interdit de supposer que certains arguments ReCEM puissent être des arguments inductifs, ce qui marquerait ici une différence entre ma position et celle d'Harman. Pour ce qui est de savoir si des arguments inductifs peuvent être valides, ou si on peut parler de logique inductive, je préfère laisser cette question en suspens ici. Néanmoins, j’aimerais insister sur l'idée qu'admettre que des arguments ReCEM puissent être inductifs ne revient pas à admettre que des raisonnements proprement dits puissent être assimilés à ces arguments. 
principalement comme nous le verrons plus loin). L'intention de Harman est en fait de renouveler notre étude du raisonnement. Il affirme:

Reasoned change in view [...] does not seem to have been studied much except for some recent research into planning and «belief revision » in the field of artificial intelligence (e.g. Doyle 1980). One possible cause of this otherwise general neglect is that reasoning in this sense may be conflated with reasoning in another sense, namely argument for, or proof of, a conclusion from premises via a series of intermediate steps. [...] Clearly, argument or proof is not at all the same sort of thing as reasoning in the sense of reasoned change in view $^{14}$.

Selon lui, il y aurait deux manières de concevoir le raisonnement: une première où le raisonnement est compris comme un processus de révision, et une deuxième où le raisonnement est compris comme un argument militant en faveur d'une conclusion découlant de prémisses (en passant par des étapes intermédiaires). Le problème qu'identifie Harman est que ces deux manières de concevoir le raisonnement ne sauraient être confondues: un argument ne correspond pas à un raisonnement compris comme un processus de révision. Mais est-ce à dire que, selon Harman, nos raisonnements n'entretiennent aucun lien avec des arguments? La réponse à cette question se trouve dans sa discussion des notions d'implication et d'inconsistance, notamment lorsqu'il se demande comment ces notions devraient intervenir dans les principes de révision que nous suivons/devons suivre. Malgré le fait que Harman insiste sur l'importance de ne pas réduire un raisonnement à un argument (militant en faveur d'une conclusion découlant de prémisses), il réserve toutefois un certain rôle à l'étude des «règles» qui gouvernent l'argument (l'implication) dans l'étude du raisonnement.

L'idée de départ d'Harman est qu'une théorie du raisonnement ne peut être réduite à une théorie logique puisqu'il existe une différence de catégorie entre les règles de l'implication (logique) et les principes de révision: les règles de l'argument ne sont pas celles que quelqu'un suit effectivement pour modifier sa propre conception. Par exemple, la règle du modus ponens dit qu'un conditionnel et son antécédent impliquent conjointement le conséquent du conditionnel. Harman remarque, à juste titre, que ce n'est sûrement pas le cas qu'à chaque fois que quelqu'un croit un conditionnel et croit aussi son antécédent, il doit ou peut rationnellement croire son conséquent puisqu'il est tout à fait plausible que cette personne croit déjà la négation du conséquent et doit alors ou cesser de croire le conditionnel, ou cesser de croire l'antécédent. Cela étant dit, Harman n'exclut pas que l'implication (ou la notion d'argument) puisse être pertinente dans l'étude du raisonnement et insiste sur le fait que si l'implication (ou l'argument) a un rôle à jouer dans cette étude, ce n'est qu'en vertu du fait que certains principes de

14. Harman, 1986, p. 3. 
révision (à l'œuvre dans nos raisonnements proprement dits) référeraient à des principes logiques (sans être eux-mêmes des principes logiques proprement dits). Son objectif est en quelque sorte de montrer que la logique ne possèderait pas, selon lui, de pertinence particulière dans l'étude du raisonnement, mais que cela n'implique pas que les notions d'implication et d'inconsistance ne jouent aucun rôle dans le processus de révision ${ }^{15}$.

\section{Implication et inconsistance, logiques et reconnues}

Ce refus de reconnaitre à la logique le rôle de théorie normative du raisonnement s'explique par la manière dont Harman conçoit l'activité de raisonner. C'est seulement en examinant ce qui caractérise le processus psychologique dans lequel l'agent est engagé qu'il sera possible, selon Harman, d'identifier les normes du raisonnement, ce que la logique ne peut réussir à capturer ${ }^{16}$. Toutefois, cela ne revient pas à dire qu'il n'existerait pas de liens de parenté entre certains principes logiques et les principes de révision qu'il compte identifier. L'intention d'Harman est ainsi d'examiner les liens entre certains principes de révision à l'œuvre dans nos raisonnements (principes du raisonnement) et certaines règles logiques (qui gouvernent les relations entre les propositions), notamment dans le but de déterminer comment des principes de révision pourraient invoquer les notions d'implication et d'inconsistance. Examinons son argument.

Il suggère d'abord que si la logique était pertinente d'une manière quelconque au raisonnement, cette pertinence devrait pouvoir être saisie (au moins grossièrement) à travers des principes de révision qui pourraient ressembler à ce qu'il nomme le principe d'implication logique (le fait que les croyances de quelqu'un impliquent logiquement $P$ peut être une raison d'accepter $P$ ) et le principe d'inconsistance logique (l'inconsistance logique doit être évitée). Pour expliquer ce qui distingue ces principes, il considère alors l'exemple suivant: supposons que quelqu'un croie à la fois $P$ et aussi si $P$ alors $Q$. Puisque les croyances de cet individu impliquent $Q$, le principe d'implication logique dit que cela peut lui donner une raison de croire $Q$;

15. «Even if they agree that logic is not by itself a theory of reasoning, many people will be inclined to suppose that logic has some sort of special relevance to the theory of reasoning. In this chapter I argue that this inclination should be resisted. It turns out that logic is not of any special relevance» (Harman, 1986, p. 11).

16. Dans Change in View, Harman indique: "My aim in this book is to contribute to the development of a theory of reasoned revision, but I find it hard to say whether the theory I want is a normative theory or a descriptive theory. A normative theory says how people ought to reason, whereas a descriptive theory says how they actually $d o$ reason. The theory I envision tries to say either or both of these things. Actually, normative and descriptive theories are intimately related. For one thing, it is hard to come up with convincing normative principles except by considering how people actually do reason, which is the province of a descriptive theory. [...] The distinction between a normative and a descriptive theory seems as clear as the thought that one might sometimes reason in a way in which one ought not to have reasoned, in which case there is something wrong with one's reasoning» (Harman, 1986, p. 7). 
mais il ne dit pas que l'agent devrait aussi s'empêcher de croire la négation de $Q$ (nonQ) (c'est ce qui distingue le principe d'implication logique du principe d'inconsistance logique): c'est-à-dire que croire non $Q$ et croire aussi $P$ et si $P$ alors $Q$ est contraire au principe d'inconsistance logique mais non au principe d'implication logique. Corollairement, il fait remarquer que le principe d'inconsistance logique ne dit pas que quelqu'un a une raison de croire $Q$ s'il croit $P$ et si $P$ alors $Q$. Harman identifie alors une première différence importante entre ces principes de révision qui ont trait au raisonnement, et la règle du modus ponens et le principe de non-contradiction, à savoir que chacun des principes de révision proposés (implication logique et inconsistance logique) est défaisable; chacun de ces principes rencontrera nécessairement des exceptions. Pour illustrer cette thèse, il considère en premier lieu le principe d'implication logique. Ce principe dit que si quelqu'un croit à la fois que $P$ et que si $P$ alors $Q$, cela peut être une raison pour lui de croire que $Q$. Mais, selon Harman, il est clair que ce n'est pas toujours une raison de croire que $Q$. En effet, il peut y avoir des cas où quelqu'un croit que $P$ et croit que si $P$ alors $Q$, mais ne devrait pas se mettre à croire (come to believe) que $Q^{17}$. L'idée d'Harman est, je crois, de mettre en évidence un type de cas où croire à la fois que $P$ et que si $P$ alors $Q$ non seulement pourrait ne pas être une raison de croire que $Q$, mais ne devrait pas être une raison de croire que $\mathrm{Q}^{18}$. Pour remédier à ce problème, il suggère alors de remplacer le principe d'implication logique par le principe de clôture logique qu'il formule de la manière suivante:

17. L'exemple d'Harman est celui de Mary qui en vient à croire trois choses inconsistantes: elle croit que si elle regarde dans le garde-manger, elle verra une boîte de Cherrios; elle regarde dans le garde-manger mais ne voit pas de boîte de Cherrios. Selon Harman, Mary ne devrait pas conclure à ce point qu'elle voit réellement une boîte de Cherrios sur la base de ses deux croyances antérieures.

18. Pourtant, cela ne contredit pas le principe d'implication logique si l'on s'en tient à la formulation de Harman. Le principe ne dit pas que «le fait que les croyances de quelqu'un impliquent logiquement $P$ doit être une raison d'accepter $P$ » mais bien que «le fait que les croyances de quelqu'un impliquent logiquement $P$ peut être une raison d'accepter $P$ ». Il admet donc des cas où le fait que les croyances de quelqu'un impliquent logiquement $P$ n'est pas une raison pour lui de croire que $P$. Il est clair que Harman veut insister ici sur le fait que ces croyances non seulement ne peuvent pas, mais qu'elles ne devraient pas être une raison de croire que $P$ : c'est-à-dire que l'existence du lien d'implication peut ne pas être une raison de croire que $P$ mais que, dans certains cas, ne devrait pas être une raison de croire que $P$. L'idée d'Harman semble être la suivante: à $t$, Mary croit qu'il y a une boîte de Cherrios dans le gardemanger $(P)$, et croit que s'il y a une boîte de Cherrios dans le garde-manger, alors elle verra une boîte de Cherrios si elle regarde dans le garde-manger (si P, alors Q). Supposons maintenant qu'il n'y ait pas de boîte de Cherrios dans le garde-manger. À $t+1$, Mary regarde dans le gardemanger mais ne voit pas de Cherrios. Dans la mesure où elle croit à la fois $P$ et si $P$ alors $Q$ à $t$, et qu'elle ne voit pas de boîte de Cherrios dans le garde-manger à $t+1$, si $P$ et si $P$ alors $Q$ pouvaient être des raisons de croire $Q$ à $t+1$, elle pourrait passer toute la matinée à déplacer et replacer ce qui se trouve dans son garde-manger dans l'espoir de trouver la boîte de Cherrios. Il semble plutôt que, du fait qu'elle croit non $Q$ à $t+1, P$ et si $P$ alors $Q$ non seulement ne 


\section{Principe de clôture logique:}

Les croyances d'un agent doivent être «closes sous l'implication logique» (closed under logical implication). En d'autres termes, il y a quelque chose de défectueux (wrong) dans les croyances d'un agent s'il y a une proposition logiquement impliquée par ces croyances que l'agent ne croit pas déjà. Dans un tel cas, l'agent doit ou bien ajouter la proposition impliquée à l'ensemble de ses croyances ou bien abandonner l'une des croyances (prémisse ?) entraînant cette implication.

Il fait alors remarquer que ce principe (tel que défini ici) est tout aussi défectueux puisque plusieurs choses triviales sont impliquées par ce que quelqu'un croit, et qu'il serait inutile de les « ajouter" à ce qu'il croit déjà ${ }^{19}$. C'est-à-dire qu'il n'y a pas de raison de s' "encombrer» l'esprit de toutes ces propositions. Ce qui le conduit à poser (et à admettre) un premier principe du raisonnement que nous suivons ou que nous devons suivre lorsque nous raisonnons, qui consiste à éviter l'encombrement (clutter avoidance): quelqu'un ne doit pas encombrer son esprit de trivialités ${ }^{20}$. Harman reconnaît

peuvent pas être des raisons de croire $Q$, mais ne devraient pas être des raisons de croire $Q$ puisque $P$, si $P$ alors $Q$ et $Q$ sont faux.

Pourtant, à première vue, on est tenu d'admettre qu'il ne s'agit que d'un sous-ensemble de cas appartenant à la classe des cas où on observe que le fait que les croyances de quelqu'un impliquent $P$ peut ne pas être une raison de croire que $P$. Si Harman veut faire de ce sousensemble de cas une classe à part, il devrait alors reformuler son principe d'implication logique (puisque ces cas ne contredisent pas le principe tel que formulé).

19. «For example, if one believes P, one's view trivially implies "either P or Q", "either $\mathrm{P}$ or P", "P and either P or R", and so on» (Harman, 1986, p. 12).

20. Selon Harman, admettre ce principe obligerait à distinguer croyances explicites et croyances implicites: il remarque qu'admettre que l'esprit de quelqu'un pourrait être encombré de croyances pourrait revenir à supposer des choses telles que: 1) ajouter à des croyances des propositions impliquées par celles-ci prend un certain temps, et que ce temps serait peut-être mieux utilisé autrement; et/ou 2) qu'un agent a une "capacité limitée d'entreposage " pour ses croyances, et qu'il y a ainsi une limite au nombre de choses que quelqu'un peut croire; et/ou 3) il y a des limites à la récupération d'information (information retrieval), et donc plus un agent a un nombre de croyances élevé, plus il sera difficile pour lui de se rappeler des croyances pertinentes au moment où il en aura besoin. Il note ensuite que ces suppositions présupposent que nos croyances seraient explicitement représentées dans l'esprit, et que nous devrions être prudents lorsqu'on affirme une telle présupposition. ( Not all one's beliefs can be explicitely represented in this way, since one could believe only finitely many things. But one can and does believe infinitely many things. For example, one believes the earth does not have two suns, the earth does not have three suns, the earth does not have four suns, and so on " (Harman, 1986, p. 12). Harman propose alors de distinguer ce que quelqu'un croit explicitement de ce quelqu'un croit implicitement. Il définit alors ce que quelqu'un croit explicitement comme suit: un agent croit quelque chose explicitement si la croyance qu'il a implique une représentation mentale explicite dont le contenu est le contenu de cette croyance. Par opposition, une chose est crue implicitement lorsqu'elle n'est pas crue explicitement mais qu'elle peut être facilement inférée à partir de ce qui est cru explicitement (à partir des croyances explicites de l'agent). (Harman discute de différents types de croyances implicites et aussi d'autres distinctions pouvant être effectuées notamment entre croyances conscientes et croyances inconscientes, entre croyances occurentes et croyances dispositionnelles (voir p. 13-14). Il évalue ensuite si le principe de 
donc le principe qui consiste à «éviter l'encombrement de son propre esprit » à la suite de son rejet du principe de clôture logique comme principe du raisonnement. Il s'agirait en fait d'un méta-principe de raisonnement dont le rôle serait de contraindre les principes de révision ${ }^{21}$. Or, il est tout à fait clair que ce principe ne correspond à aucun principe logique.

Après avoir montré que le principe d'implication logique ne saurait s'appliquer au raisonnement sans que ne surgissent des exceptions, il indique ensuite que des remarques similaires s'appliquent au principe d'inconsistance logique (selon lequel un agent doit éviter l'inconsistance). L'exemple est le suivant: il arrive parfois que quelqu'un découvre que ses croyances sont inconsistantes sans savoir comment il pourrait les réviser pour lever l'inconsistance, sans que cela ne soit coûteux pour lui. Dans un tel cas, la meilleure stratégie qui s'offre à l'agent peut être de maintenir l'inconsistance et d'essayer d'éviter des raisonnements qui font intervenir les croyances en cause. Selon Harman, ce genre de situation surgit dans la vie de tous les jours, notamment lorsque quelqu'un n'a pas le temps d'arriver à savoir ce qu'il devrait faire avec une inconsistance qu'il vient de découvrir. Il conclut que, dans un tel cas, il est rationnel de simplement maintenir les croyances contradictoires en essayant de ne pas exploiter l'inconsistance en cause ${ }^{22}$. Cet argument sur lequel Harman s'appuie pour rejeter le principe d'inconsistance logique permet de mettre en lumière l'aspect normatif de sa théorie du raisonnement. L'intention d'Harman est d'identifier à quelles conditions

clôture logique selon lequel les croyances d'un agent devraient être closes sous l'implication logique s'applique aux croyances explicites. Il montre alors que ce principe ne s'applique pas dans la mesure où un agent possède seulement un nombre fini de croyances explicites et que celles-ci ont plusieurs conséquences logiques (à l'infini). Il montre ensuite que ce principe ne s'applique pas plus aux croyances implicites: on ne peut pas attendre de quelqu'un qu'il croie, même implicitement, la conséquence logique d'une de ses croyances si une preuve complexe est nécessaire pour voir l'implication. Harman montre ensuite que même une version plus faible du principe de clôture logique - notamment que les croyances d'un agent doivent être closes sous l'implication logique évidente - ne pourrait s'appliquer au raisonnement. ( It won’t help to change the Logical Closure Principle to say one's beliefs should be closed under obvious logical implication. That would come to the same thing, since any logical implication can eventually be demonstrated by a proof consisting entirely of a series of obvious steps. This means that, if beliefs are required to be closed under obvious logical implication, they are required to be closed under any logical implication, obvious or not. So, since beliefs cannot be required to be closed under logical implication, they cannot be required to be closed under obvious logical implication either» (Harman, 1986, p. 14).

21. "The principles of revision must be such that they discourage a person from cluttering up either long-term memory or short-term procesing capacities with trivialities. One way to do this would be to allow one to accept a new belief $P$ only if one has (or ought to have) an interest in whether P is true» (Harman, 1986, p. 15).

22. Harman indique que ce genre de situation peut aussi se produire sur le plan de la réflexion, par exemple lorsque quelqu'un croit que toutes ses croyances ne peuvent pas être vraies: dans un tel cas, la personne peut être tout à fait justifiée de continuer à croire cela et de croire (aussi) chacune de ses autres croyances. Ce problème (discuté par Foley) concerne le fait de savoir s'il est possible d'être rationnel et épistémiquement inconsistant. 
les croyances et les actions des agents peuvent être considérées comme rationnelles tout en montrant que la rationalité des croyances et les actions des agents est indépendante du fait qu'ils suivent des principes logiques. (Les principes de révision auxquels Harman réfère correspondraient en fait aux normes de rationalité qu'il cherche à identifier.) Ayant jusqu'ici démontré que les principes d'implication et d'inconsistance logique ne pouvaient être des principes de révision en vertu du fait qu'ils souffriraient nécessairement d'exceptions, Harman propose de montrer que cela n'implique pas que les notions d'implication et d'inconsistance n'ont aucun rôle à jouer dans le raisonnement, et il étaye sa thèse en recourant aux notions d'implication immédiate et d'inconsistance immédiate. Ainsi, s'il admet que la logique est peu pertinente pour le raisonnement, ces deux notions y joueraient, par contre, un rôle particulier dans le raisonnement.

Reprenant le problème posé par le principe d'implication logique, Harman rappelle que quelqu'un peut n'avoir aucune raison de croire ce qui est logiquement impliqué par ses croyances s'il ne dispose pas d'un argument simple et court permettant de montrer l'existence de ce lien d'implication $^{23}$. Il suggère alors de remplacer le principe de l'implication logique (et le principe de l'inconsistance logique) par deux nouveaux principes:

Principe de l'implication logique reconnue:

Quelqu'un a une raison de croire $P$ s'il reconnaît que $P$ est logiquement impliqué par ce qu'il croit.

\section{Principe de l'inconsistance logique reconnue:}

Quelqu'un a une raison d'éviter de croire des choses qu'il reconnait être logiquement inconsistantes.

Mais, selon Harman, un nouveau problème découle alors de ces nouveaux principes: on ne peut reconnaître une implication logique ou une inconsistance logique qu'à condition de posséder le concept d'implication logique ou d'inconsistance logique, et il fait remarquer que peu de gens possèdent de tels concepts, du moins si cela implique qu'ils soient capables de distinguer l'implication et l'inconsistance logique d'autres sortes d'implication et d'inconsistance ${ }^{24}$. Il en conclut que les principes de l'implication

23. Il illustre cette idée par l'exemple suivant: "To take an extreme example, one accepts basic principles of arithmetic that logically imply some unknown proposition $P$ which is the answer to an unsolved mathematical problem: but one has no reason to believe $P$ if one is not aware that $\mathrm{P}$ is implied by these principles " (Harman, 1986, p. 17).

24. Il illustre cette idée comme suit: "Consider the following examples:

$P$ or $Q$ and not $P$ taken together imply $Q$.

$A=B$ and $B=C$ taken together imply $A=C$.

$A<B$ and $B<C$ taken together imply $A<C$.

$A$ is part of $B$ and $B$ is part of $C$ taken together imply $A$ is part of $C$.

$X$ is Y's brother implies $X$ is male.

Today is Thursday implies Tomorrow is Friday. 
logique reconnue et de l'inconsistance logique reconnue ne possèderaient qu'une application limitée, c'est-à-dire ne pourraient, à proprement parler, constituer des principes ou des règles de révision du raisonnement. Pour contrer cette objection, il propose de généraliser ces principes, c'est-à-dire de supprimer la mention spécifique d'implication logique et d'inconsistance logique, en reformulant les principes comme suit:

Principe de l'implication reconnue:

Quelqu'un a une raison de croire $P$ s'il reconnaît que $P$ est impliqué par ce qu'il croit (one's view).

Principe de l'inconsistance reconnue:

Quelqu'un a une raison d'éviter de croire des choses qu'il reconnait être inconsistantes.

Admettant que ces principes ne pourraient s'appliquer qu'à des agents possédant les concepts d'implication et d'inconsistance, il considère que la formulation de ces principes est moins problématique, puisque (selon lui) la possession de ces concepts serait moins exigeante. Il en conclut donc:

1) que ces principes seraient suffisants pour montrer que lorsque nous raisonnons (reasoned changes in one's view), nous sommes d'une certaine manière sensibles à l'implication et à l'inconsistance ${ }^{25}$;

2) que lorsqu'un agent serait disposé à traiter ses croyances que $P$ et que si $P$ alors $Q$ comme des raisons de croire que $Q$, il posséderait, en vertu de cette disposition, une habileté à reconnaître cette sorte d'implication (le même raisonnement valant pour l'inconsistance);

3) et que des agents pourraient, par conséquent, posséder certaines dispositions reflétant leurs habiletés à reconnaître certaines sortes d'implication et certaines sortes d'inconsistance ${ }^{26}$.

Ces conclusions permettent de préciser le rôle que Harman veut attribuer à l'implication et à l'inconsistance dans l'étude du raisonnement: il se déclare enclin à considérer fondamentales certaines dispositions que des agents auraient à traiter les propositions de certaines manières, notamment des dispositions à traiter certaines propositions comme en impliquant

X plays defensive tackle for the Philadelphia Eagles implies X weighs more than 150 pounds. People who recognize these and related implications do not in any consistent way distinguish purely logical implications and others that are not purely logical» (Harman, 1986, p. 17-18).

25. "I suggest it is enough to be able to make reasoned change in one's view in a way that it is sensitive to implication and inconsistency" (Harman, 1986, p. 18).

26. "Someone who is disposed to treat beliefs in $P$ and if $P$ than $Q$ as reasons to believe $Q$ has, by virtue of that very disposition, an appropriate ability to recognize this sort of implication, at least if this disposition is also accompanied by the disposition not to believe $P$, if $P$ than $Q$, and not $Q$. And the latter sort of disposition might reflect an appropriate ability to recognize that sort of inconsistency" (Harman, 1986, p. 18). 
d'autres de manière immédiate, et d'autres comme étant inconsistantes entre elles de manière immédiate. Ce qui le conduit à admettre comme principes fondamentaux:

$P, Q, \ldots, R$ impliquent immédiatement $S$ pour $A$

$P, Q, \ldots, R$ sont immédiatement inconsistantes pour $A$

Il soulève ensuite la question de savoir si ces notions de base pourraient être réduites à d'autres notions (notamment à celle de raisons). Il soutient que non: différentes choses nous interdisent d'admettre que les propositions $« P$, $Q, \ldots, R$ impliquent immédiatement $S$ pour $A$ » et « $A$ est disposé à traiter $P$, $Q, \ldots, R$ comme une raison de croire $S$ » sont équivalentes. Il justifie cette idée en indiquant que, par exemple, dans certains cas particuliers, d'autres considérations pourraient l'emporter sur la disposition générale de $A$, notamment si $S$ est absurde. Dans un tel cas, certaines croyances de $A$ impliqueraient immédiatement une proposition pour $A$, même si $A$ ne serait pas disposé à traiter ces croyances comme des raisons de croire cette proposition ${ }^{27}$. Il ajoute que cette impossibilité d'assimiler ces deux dispositions (la disposition de $A$ à traiter certaines croyances comme en impliquant immédiatement une autre et la disposition de $A$ à traiter ces croyances comme des raisons de croire cette autre) est d'autant plus justifiée que $A$ pourrait aussi traiter certaines de ses croyances comme des raisons de croire une conclusion sans qu'il n'ait à considérer ces croyances comme impliquant immédiatement cette conclusion. Il conclut ainsi :

So, I am inclined simply to assume one has certain basic dispositions to take some propositions immediately to imply other propositions and to take some propositions as immediately inconsistent with each other. More generally, I assume one can have general dispositions with respect to certain patterns of immediate implication and inconsistency even if some instances of the patterns are so long complex or otherwise distracting that one has no particular disposition to take those particular instances to be immediate implications or inconsistencies $^{28}$.

Ces remarques concernant le rôle qui doit être attribué aux «règles» gouvernant l'argument (l'implication) dans l'étude du raisonnement sont, à mon avis, suffisantes pour saisir la place que Harman veut accorder à la logique et à l'argument dans son analyse. Résumons. Selon lui, il n'y a pas de manière significative où la logique est pertinente pour le raisonnement. Néanmoins, certains principes de révision (raisonnement) ont des liens de parenté avec certains principes logiques. Bien que les principes de l'implica-

27. On pourrait néanmoins admettre que, parmi les raisons qu'un agent a de croire quelque chose, certaines sont concluantes et d'autres non. Dans ce cas-ci, les croyances de A qui impliquent immédiatement une proposition pour A pourraient être des raisons de croire cette proposition mais ne seraient pas concluantes. Inversement, les autres considérations qui l'emporteraient sur la disposition générale de $\mathrm{A}$ seraient, elles, des raisons concluantes.

28. Harman, 1986, p. 19. 
tion et de l'inconsistance logique ne soient pas applicables au raisonnement, cela n'entraîne pas, selon Harman, que l'implication et l'inconsistance n'aient pas de rôle à jouer dans le raisonnement, c'est-à-dire dans le processus de révision (d'où le lien de parenté des principes de révision avec certains principes logiques ${ }^{29}$. Pour rendre compte du rôle de l'implication et de l'inconsistance dans le raisonnement, Harman admet deux principes du raisonnement: les principes de l'implication reconnue et de l'inconsistance reconnue. La possibilité pour un agent de reconnaître une implication immédiate et une inconsistance immédiate serait liée au fait qu'il possède une certaine disposition par rapport à des propositions: lorsqu'un agent possède une sorte d'habileté à traiter ses croyances que $P$ et que si $P$ alors $Q$ comme des raisons de croire que $Q$, il peut alors être disposé à reconnaître une implication immédiate ou une inconsistance immédiate (il peut être disposé à traiter ces croyances comme des raisons de croire que Q). Ce qui ne revient pas à dire que le fait d'être disposé à traiter certaines croyances comme en impliquant une autre soit équivalent au fait de traiter ces croyances comme des raisons de croire cette autre: différents cas permettent de montrer que même si un agent est disposé à reconnaître que certaines propositions impliquent immédiatement une autre proposition, il est possible que l'agent ne soit pas disposé à traiter ces propositions comme des raisons de croire celle qui est impliquée ${ }^{30}$. Ces précisions concernant l'analyse de Harman nous seront utiles pour déterminer si sa théorie permet d'expliquer adéquatement ce qu'est un raisonnement. Nous sommes maintenant en mesure de revenir à la question soulevée au début.

\section{Le processus compris de manière externe et la perspective de la première personne}

J'ai proposé qu'une étude du raisonnement nous oblige à admettre: 1) que les raisonnements sont des processus qui mettent en relation des états mentaux ayant des contenus propositionnels; 2) que les raisonnements se distinguent des arguments qui mettent en relation les contenus propositionnels de certains des états mentaux impliqués dans des raisonnements proprement dits (c'est-à-dire, les arguments ReCEM) ; 3) qu'on ne peut faire abstraction du rôle que jouent ces arguments dans les raisonnements proprement dits, notamment pour expliquer la relation entre les aspects processuel et inférentiel du raisonnement.

29. Lorsque Harman indique que nous ne raisonnons pas déductivement, c'est en vertu du caractère défaisable des principes de l'implication et de l'inconsistance logique, ce qui ne revient pas, selon lui, à dire que les raisonnements ne sont pas sensibles à l'implication et à l'inconsistance.

30. Comme nous l'avons vu plus haut, Harman semble assimiler le concept de raison à celui de raison concluante. 
Nous avons vu que, de son côté, Harman conçoit un raisonnement comme un "reasoned change in view", et que son étude vise précisément à élucider (1): il s'intéresse au caractère processuel du raisonnement, au raisonnement lui-même, à ce qui se passe lorsqu'un agent passe d'un ensemble d'états mentaux à $t$ à un nouvel ensemble d'états mentaux à $t+1$. Nous avons tenté de déterminer si Harman réservait un rôle aux arguments dans son étude du raisonnement (notamment aux arguments ReCEM), ou en d'autres termes s'il s'intéresse à élucider (3). De prime abord, on aurait pu être tenté de conclure que non, vu son intention de limiter le rôle de la logique dans l'étude du raisonnement, et de refuser qu'un raisonnement puisse être assimilé à un argument. J'ai néanmoins indiqué que cela n'était pas suffisant pour en arriver à cette conclusion dans la mesure où admettre qu'un raisonnement n'est pas un argument et choisir de limiter le rôle de la logique dans l'étude du raisonnement ne revient pas à nier qu'un argument puisse être associé au raisonnement proprement dit d'un agent.

La réponse à cette question (celle de savoir si Harman réserve un rôle aux arguments ReCEM dans son étude du raisonnement) dépend de la question de savoir en quoi consiste ce processus de révision dans lequel est engagé celui qui raisonne. Harman veut proposer une théorie du raisonnement qui concerne l'activité même de raisonner (reasoned change in view): il veut expliquer, d'un point de vue externe, ce qui se passe dans la tête de l'agent lorsqu'il raisonne. Le raisonnement y est conçu comme un processus de révision, son intention étant de montrer qu'expliquer comment on raisonne consiste à expliquer le passage d'un ensemble d'états mentaux à un nouvel ensemble d'états mentaux (le passage de $t$ à $t+1$ ). Néanmoins, même si le raisonnement peut être conçu, d'un point de vue externe, comme un processus tel qu'Harman le suggère, il peut aussi être conçu comme l'activité de l'agent qui raisonne. Ainsi, en réduisant l'activité de raisonner à un processus en quelque sorte "impersonnel ", Harman est conduit à confondre (ou à réduire) ce que fait l'agent qui raisonne (le fait que raisonner soit une activité de l'agent) et ce qui se passe dans sa tête (le processus) pendant qu'il raisonne.

J'aimerais insister sur le fait que ces deux manières de concevoir l'activité de raisonner sont différentes mais indissociables. Expliquer ce qui se passe dans la tête de l'agent lorsqu'il raisonne consiste à expliquer comment l'agent est passé d'un ensemble d'états mentaux à $t$ à un nouvel ensemble d'états mentaux à $t+1$ : le raisonnement est alors compris comme une mise en relation d'états mentaux possédant des contenus propositionnels. Cela concerne la relation entre le raisonnement de l'agent et la rationalité de ses attitudes. Expliquer ce que l'agent fait lorsqu'il raisonne consiste à se mettre à la place de l'agent qui met en relation les contenus propositionnels de certains de ses propres états mentaux. Cela concerne, cette fois, la relation entre le raisonnement proprement dit de l'agent et ses «raisons ». Lorsqu'on s'intéresse, comme Harman, au raisonnement de l'agent d'un point de vue 
externe, on cherche principalement à savoir comment les raisonnements peuvent contribuer au fait que des agents peuvent être considérés comme rationnels: on s'intéresse à une question normative qui concerne le fait d'identifier les principes de révision que suit/doit suivre celui qui raisonne correctement.

Lorsqu'on s'intéresse au raisonnement du point de vue de ce que fait l'agent pendant qu'il raisonne, il est clair qu'on adopte un point de vue différent: au-delà de concevoir le raisonnement comme un simple processus pouvant être analysé d'un point de vue externe, on le conçoit comme une activité faite par l'agent qui, dans la perspective de l'agent qui raisonne, met en relation non pas (certains de) ses états mentaux mais les contenus propositionnels mêmes de certains de ses états mentaux (ce qui met en lumière l'aspect inférentiel du raisonnement). Je suggère de nommer cette deuxième manière de concevoir le processus «la perspective de la $1^{\text {re }}$ personne». Mais, adopter la "perspective de la $1^{\text {re }}$ personne» ne revient pas à assimiler le raisonnement proprement dit de l'agent à un argument dont la conclusion découle de prémisses (en passant par des étapes intermédiaires) puisque l'argument ReCEM de l'agent n'est pas le raisonnement proprement dit de l'agent, c'est-à-dire le raisonnement qu'il fait (à la première personne). Il est ainsi possible de concevoir ce processus de révision de manière externe (ce que fait Harman), mais aussi du point de vue même de la personne qui est engagée dans ce processus (perspective de la $1^{\text {re }}$ personne): cela est précisément lié au double aspect (processuel et inférentiel) spécifique au raisonnement. Par conséquent, si les liens entre certains arguments et les raisonnements proprement dits des agents méritent d'être étudiés, c'est dans la mesure où ils promettent de nous renseigner sur ce qui lie ces aspects processuel et inférentiel, ce dont une théorie du raisonnement devrait être en mesure de rendre compte. C'est-à-dire que, dans la mesure où des arguments peuvent représenter les contenus de certains des états mentaux impliqués dans des raisonnements proprement dits (ReCEM), cela explique pourquoi ils doivent être pris en considération dans l'étude du raisonnement, notamment en vertu du fait qu'ils sont justement liés à la perspective de la $1^{\text {re }}$ personne.

Ce n'est pas un hasard si les raisonnements proprement dits des agents ont souvent été confondus avec des arguments: lorsqu'on demande à un agent «Pourquoi crois-tu que $x$ ? » et qu'il répond "parce que $y$ et si $y$ alors $x$ ", nous considérons que $y$ et si $y$ alors $x$ sont en quelque sorte ses raisons de croire que $x$, et que sa conclusion n'est pas indépendante du fait qu'il ait raisonné. Supposons que l'on tente de représenter le raisonnement de l'agent à l'aide d'un argument, on risque alors d'obtenir: « « $y$ », «si y alors $x$ » donc « $x$ » . J'ai mentionné que le problème traditionnel auquel a été confrontée l'étude du raisonnement avait été celui de concevoir l'activité de raisonner comme une enquête interrogative de type argumentative, qui a conduit en quelque sorte à ne prendre en considération que l'aspect «inférentiel » du raisonnement. Je crois que c'est précisément ce que Harman veut éviter en 
dissociant les notions de raisonnement et d'argument, en voulant insister sur son aspect psychologique. Il veut rendre compte de ce qui se passe à l'intérieur de l'agent, mais en se plaçant d'un point de vue externe: il ne semble pas s'intéresser à la manière dont nous rendons compte de nos propres raisonnements (perspective à la $1^{\text {re }}$ personne), au fait qu'un agent puisse référer au contenu de certains de ses états mentaux à l'aide d'un argument, ni à la relation qui existe entre le fait que nous raisonnions et la manière dont nous en rendons compte. La question est ainsi celle de savoir si concevoir, comme Harman le fait, le processus de révision d'un point de vue externe conduit, à l'inverse, à ignorer cet aspect inférentiel qui est, lui aussi, propre au raisonnement.

Le point sur lequel j'aimerais maintenant insister est que, dès le moment où Harman choisit d'accorder un rôle aux notions d'implication reconnue et d'inconsistance reconnue dans l'explication de ce processus qui consiste à passer d'un ensemble d'états mentaux à $t$ à un nouvel ensemble d'états mentaux à $t+1$, il donne une nouvelle direction à son étude du raisonnement (reasoned change in view). Du moment où il indique que des agents peuvent avoir certaines dispositions à reconnaître une implication immédiate (c'est-à-dire qu'ils peuvent, selon sa conception, être disposés à traiter les croyances que $P$ et que si $P$ alors $Q$ comme des raisons de croire que $Q$ ), il ne s'intéresse plus simplement à expliquer ce processus de manière externe mais fait intervenir le point de vue de l'agent qui raisonne, correspondant à la perspective de la $1^{\text {re }}$ personne. Par conséquent, il semble qu'Harman n'ait d'autre choix que de s'intéresser aussi, au moins indirectement, à l'argument $\mathrm{ReCEM}$ auquel un agent a recours pour exprimer son raisonnement proprement dit (ce qui équivaut à la thèse 2). J'ai soutenu que ces arguments ReCEM revêtent une importance toute particulière dans une étude du raisonnement et que nous ne pourrions en faire abstraction (ce qui équivaut à la thèse 3). Nous sommes maintenant en mesure de voir que l'importance qui doit être accordée à ces arguments repose en fait sur celle qui doit être accordée à la perspective de la $1^{\text {re }}$ personne, et que même si Harman s'intéresse principalement au processus de révision d'un point de vue externe, les conclusions auxquelles il arrive lorsqu'il traite d'implication et d'inconsistance montrent qu'il ne peut faire l'économie de la perspective de la $1^{\text {re }}$ personne, bien qu'il ne le reconnaisse pas explicitement. Compte tenu de cette conclusion, et dans la mesure où j'ai admis avec Harman qu'un raisonnement est un processus psychologique, que raisonner ne consiste pas à suivre des principes logiques, ou encore qu'un raisonnement n'est pas un argument, on pourrait être tenté de considérer que le rôle attribué ici à la perspective de la première personne est essentiellement complémentaire au point de vue d'Harman, dans la mesure où la conception qu'il a du processus de révision ne conduit pas pour autant à nier l'aspect inférentiel du raisonnement (comme l'illustrent les principes d'implication reconnue et d'inconsistance reconnue qui prennent en considération la perspective de la $1^{\text {re }}$ personne). J'aimerais 
montrer pourquoi une telle suggestion serait erronée et esquisser sommairement certaines confusions et difficultés auxquelles conduit son approche.

\section{Ce que révèle la perspective de la première personne}

J'ai admis que lorsqu'un agent donne les raisons pour lesquelles il croit que $\mathrm{x}$, il peut le faire au moyen d'un argument. Une des questions centrales à élucider, que font apparaître la distinction raisonnement/argument ReCEM et la perspective de la $1^{\text {re }}$ personne, est celle de savoir quelle est la nature du lien qui existe entre cet argument, les raisons de l'agent et son raisonnement proprement dit. Cette question est relativement complexe, et mon intention n'est que d'esquisser sommairement ce qu'elle met en cause. Précisons d'entrée de jeu qu'elle renvoie implicitement à une autre question, celle de savoir comment traiter l'asymétrie normative qui peut surgir entre les normes du raisonnement et les normes épistémiques/pratiques.

Je suggère ainsi d'examiner ce que l'argument ReCEM dont il a été question depuis le début a de particulier. D'abord, il met en relation des propositions, qui sont les contenus propositionnels de certains des états mentaux impliqués dans le raisonnement proprement dit de l'agent, et qui, par opposition aux états mentaux de l'agent, sont liées quant aux prémisses et à la conclusion. Dans la mesure où la conclusion de l'argument ReCEM est une proposition (c'est-à-dire le contenu propositionnel d'un état mental), cela indique qu'une attitude propositionnelle ne peut être la conclusion d'un argument ReCEM. Par exemple, lorsqu'un argument ReCEM représente le contenu de certains états mentaux impliqués dans un raisonnement théorique proprement dit, c'est le contenu propositionnel d'une croyance, et non la croyance elle-même, qui est la conclusion de l'argument (par opposition au raisonnement proprement dit dont la «conclusion"serait la croyance nouvellement acquise ou abandonnée). Cette idée indique un premier désaccord avec la position d'Harman. Voyons pourquoi.

À l'instar de ce dernier, j'ai admis que le raisonnement de l'agent ne peut être assimilé à un argument, mais il est clair que les raisons qu'il invoque ne sont pas celles qui devraient le conduire à la nécessité d'établir cette distinction. Apparemment, cette confusion découle de la perspective à la $3^{\mathrm{e}}$ personne à laquelle sa conception du raisonnement semble vouloir se confiner. Du fait qu'il s’intéresse spécifiquement à l'aspect processuel (à la $3^{\mathrm{e}}$ personne) du raisonnement, il considère qu'un raisonnement n'est pas un argument parce qu'aucun argument ne pourrait rendre compte de l'ensemble des états mentaux impliqués au cours de ce processus: il présuppose que si un raisonnement pouvait être traduit par un argument, les prémisses de l'argument coïncideraient alors avec les états mentaux mêmes de l'agent. Cette suggestion est pour le moins paradoxale. Il est clair qu'un argument ne met pas en relation les états mentaux d'un agent: il peut, au mieux, mettre en relation certains contenus propositionnels des états mentaux impliqués dans le raisonnement proprement dit de l'agent puisqu'il s'agit d'une relation 
entre des propositions, et qu'un agent ne saurait simultanément avoir à l'esprit les contenus propositionnels de tous ses états mentaux. Par conséquent, aucun argument ne pourra jamais rendre compte de cet aspect processuel spécifique au raisonnement. C'est ce qui explique pourquoi un raisonnement n'est pas un argument. Néanmoins, dans la mesure où Harman admet aussi la nécessité de distinguer raisonnement et argument, ce désaccord sera peut-être jugé mineur. J'aimerais examiner un deuxième désaccord qui, cette fois, est plus important: il concerne la manière dont Harman conçoit ce en quoi consiste «être une raison» pour un agent.

Nous avons vu que l'intérêt porté au rôle que les arguments ReCEM ont par rapport aux raisonnements proprement dits des agents est lié à l'idée que ce que les agents prennent en considération lorsqu'ils raisonnent, ce sont les contenus propositionnels de certains des états mentaux qui participent à leur raisonnement proprement dit, lesquels correspondent aux prémisses de l'argument ReCEM (l'argument apparaît lorsqu'on se met à la place de l'agent qui raisonne). Les agents peuvent ainsi exprimer leurs raisonnements à l'aide d'arguments. C'est-à-dire qu'ils peuvent citer les raisons pour lesquelles ils croient ce qu'ils croient. J'aimerais montrer que ce qu'ils citent alors sont les prémisses de l'argument ReCEM (les contenus propositionnels de certains des états mentaux impliqués dans le raisonnement proprement dit) et que c'est précisément ce que met en lumière la perspective de la $1^{\text {re }}$ personne. Prenons un exemple pour illustrer cette idée.

Supposons l'argument A suivant:

Tous et seulement les oxoniens ont publié un livre chez Oxford.

Sam n'est pas un oxonien,

donc

Sam n'a pas publié de livre chez Oxford.

Supposons que l'argument A soit un argument correct (sound): les prémisses sont vraies, et, l'argument étant valide, alors la conclusion est nécessairement vraie.

Supposons maintenant qu'Anna croie que «tous et seulement les oxoniens ont publié un livre chez Oxford », croie que "Sam est un oxonien », qu'on lui demande si elle croit que Sam a publié un livre chez Oxford, et qu'elle réponde que oui. L'argument ReCEM que l'on peut associer au raisonnement d'Anna pourrait alors avoir la forme suivante:

Argument B:

Tous et seulement les oxoniens ont publié un livre chez Oxford U. Press. Sam est un oxonien, donc

Sam a publié un livre chez Oxford.

Supposons que nous demandions maintenant à Anna pourquoi elle croit que Sam a publié un livre chez Oxford, et qu'elle réponde parce que 
«Tous et seulement les oxoniens ont publié un livre chez Oxford» et «Sam est un oxonien ». Dans un tel cas, il semble qu'Anna considère que: "Tous et seulement les oxoniens ont publié un livre chez Oxford» et «Sam est un oxonien» lui permettent de conclure que "Sam a publié un livre chez Oxford ", c'est-à-dire que ces contenus propositionnels sont les raisons qu'elle a de croire que "Sam a publié un livre chez Oxford». Ce qui laisse entrevoir que les prémisses de cet argument correspondent aux raisons qu'elle a de croire ce qu'elle croit et non aux raisons pour lesquelles Sam a publié un livre chez Oxford. (Il n'y a pas de telles raisons puisque nous avons admis que Sam n'est pas un oxonien.)

À l'inverse, quelqu'un pourrait vouloir suggérer que, du fait qu'elle croit que "Tous et seulement les oxoniens ont publié un livre chez Oxford" et qu'elle croit que "Sam est un oxonien", elle croit que "Sam a publié un livre chez Oxford", c'est-à-dire que ce sont ses croyances (ses états mentaux, le fait qu'elle croit et non ce qu'elle croit) qui constituent les raisons qu'elle a de croire que "Sam a publié un livre chez Oxford». N'est-ce pas l'idée d'Harman lorsqu'il affirme que, lorsqu'un agent est disposé à traiter ses croyances que $P$ et que si $P$ alors $Q$ comme des raisons de croire que $Q$, il possède, en vertu de cette disposition, une habileté à reconnaître cette sorte d'implication?

Pour rendre plausible cette idée, quelqu'un pourrait suggérer d'inclure, au sein même de la proposition, un opérateur traduisant le fait qu'il s'agit d'une croyance de l'agent. Par exemple, on pourrait exprimer l'argument d'Anna comme suit (où $C$ est un opérateur exprimant «croit que»): \{[Anna $C$ «Tous et seulement les oxoniens ont publié un livre chez Oxford»], [Anna $C$ «Sam est un oxonien»] donc [Anna $C$ «Sam a publié un livre chez Oxford»]\}. Mais sans contredit, cet argument (à la $3^{\text {e }}$ personne) ne ferait pas l'affaire: ce n'est pas celui d'Anna et, de plus, il n'est pas valide. Évaluons une autre formulation qui pourrait, au yeux de certains, rendre mieux compte de l'argument associé à son raisonnement :

[Je $C$ «Tous et seulement les oxoniens ont publié un livre chez Oxford»], [Je $C$ «Sam est un oxonien»]

donc

[Je $C$ «Sam a publié un livre chez Oxford»]

Que dire de cet argument? Serait-il plus plausible? Permettrait-il de rendre compte du fait que ce sont les croyances mêmes de l'agent qui correspondent aux raisons qu'il a de croire ce qu'il croit? J'ai admis plus haut que la perspective de la première personne met en lumière un point important. Lorsqu'Anna raisonne, on peut concevoir son raisonnement proprement dit, de manière externe, comme le processus qui la conduit à acquérir un nouvel ensemble d'états mentaux. Mais, du point de vue d'Anna qui fait le raisonnement, ce ne sont pas ses états mentaux (ses croyances) qu'elle met en relation (ses croyances), mais bien leurs contenus (ce qu'elle croit). 
Revoyons maintenant l'argument à la lumière de cette idée. Devrionsnous interpréter les prémisses [Je $C$ «Tous et seulement les oxoniens ont publié un livre chez Oxford»], [Je C «Sam est un oxonien»] comme étant les raisons pour lesquelles Anna croit que "Sam a publié un livre chez Oxford"? J'ai suggéré que les prémisses de l'argument ReCEM représentent les contenus propositionnels de certains des états mentaux d'Anna (et non ses états mentaux eux-mêmes). Si cela est juste, nous devons alors admettre que [Je $C$ "Tous et seulement les oxoniens ont publié un livre chez Oxford»] est aussi le contenu propositionnel d'une croyance d'Anna, soit le contenu propositionnel d'une croyance de $2^{e}$ ordre qu'elle a. Anna croit qu' "elle croit que tous et seulement les oxoniens ont publié un livre chez Oxford». Si l'argument suggéré ne permet pas de montrer que les croyances mêmes d'Anna sont les raisons qu'elle a de croire que Sam a publié un livre chez Oxford, c'est parce qu'il exprime un autre raisonnement d'Anna qui porte sur ses croyances de $1^{\text {er }}$ ordre (ce qu'un agent peut faire aussi). Les prémisses de cet autre argument sont les raisons (non suffisantes) qu'elle a de croire "qu'elle croit que Sam a publié un livre chez Oxford", et non ses raisons de croire "que Sam a publié un livre chez Oxford». Cet argument ReCEM (qui exprime un autre raisonnement) ne porte donc pas sur les contenus propositionnels des croyances du raisonnement initial d'Anna. Les propositions [Je $C$ «Tous et seulement les oxoniens ont publié un livre chez Oxford»] et "Tous et seulement les oxoniens ont publié un livre chez Oxford» sont les contenus propositionnels de deux croyances différentes qu'a Anna, la première proposition étant le contenu propositionnel d'une de ses croyances de $2^{\mathrm{e}}$ ordre. L'ajout de [Je $C$ «..»] ne permet donc pas de montrer que les raisons qu'Anna a de croire que «Sam a publié un livre chez Oxford» sont ses croyances elles-mêmes (le fait qu'elle croit que). Il est ainsi plus plausible d'admettre que ce sont les prémisses d'un argument ReCEM qui correspondent aux raisons qu'un agent a de croire ce qu'il croit. Je suggère ainsi d'admettre que, dans la mesure où les états mentaux d'un agent ne peuvent pas être ses raisons (à moins d'être les contenus propositionnels d'états mentaux d'ordre supérieur), seuls les contenus propositionnels de certains des états mentaux impliqués dans le raisonnement proprement dit pourront assumer ce rôle.

Inversement, selon Harman, ce sont les états mentaux mêmes des agents qui lui fournissent les raisons d'acquérir ou d'abandonner d'autres états mentaux (ce sont ses croyances que $P$ et que si $P$ alors $Q$ qui constituent ses raisons de croire que Q). Il semble ainsi que si, du point de vue externe, on pourrait être tenté de supposer que les raisons qu'un agent a de croire quelque chose sont ses autres croyances, le point de vue interne (la perspective de la $1^{\text {re }}$ personne) fait apparaitre clairement que les raisons de l'agent ne peuvent pas être ses croyances mais bien les contenus de celles-ci ${ }^{31}$. Ce point est tout à

31. Si Harman concevait le processus non seulement de manière externe mais aussi du point de vue de la $1^{\text {re }}$ personne, il remarquerait que le principe de révision d'implication reconnue 
fait central: l'agent ne raisonne pas à propos de ses états mentaux mais avec le contenu de ses propres états mentaux, c'est-à-dire à la première personne. Les raisons qu'il a d'acquérir ou d'abandonner certains états ne sont pas d'autres états, mais bien les contenus de ces autres états. C'est précisément ce que ne peut pas mettre en lumière une théorie du raisonnement où le processus de révision est conçu principalement de manière externe, tel qu'Harman le suggère, et qui obscurcit substantiellement notre compréhension de ce en quoi consiste «être une raison" pour un agent.

Une théorie normative du raisonnement concerne la question de savoir comment nous pouvons déterminer rationnellement ce que nous croyons/ faisons (quelles sont les normes que doit respecter le raisonnement correct). Mais il peut arriver qu'au terme d'un raisonnement ce que l'on a identifié ne corresponde pas à ce qu'une théorie de la justification épistémique/pratique pourrait identifier comme ce que l'on devrait croirel faire (aux normes épistémiques et pratiques avec lesquelles devraient s'accorder nos croyances/nos actions), et cela même si le processus en question était parfaitement rationnel, c'est-à-dire même si nous avons raisonné correctement. Cette "asymétrie " normative n'est pas indépendante de la question de savoir comment caractériser la relation qui existe entre les « raisons » qu'un agent a de croire/d'agir, et la «rationalité » de ses croyances/actions. Ces concepts sont (du moins en partie) liés à la capacité de "raisonner» des êtres humains, et au rôle que l'on choisit d'attribuer à cette capacité. Par exemple, selon certains, raisonner donnerait à l'être humain non seulement la possibilité (i) de déterminer ce qu'il est rationnel de croire/faire, mais aussi (ii) de déterminer ce qu'il doit croire/faire, c'est-à-dire de connaître ce qui est vrai/ce qui est juste ou correct. Si l'on suit ce point de vue, l'étude proprement dite du raisonnement se trouverait précisément à l'intersection de deux aspects de la normativité: les normes qui gouvernent ce type de processus mentaux, d'une part, et les normes qui règlent les relations entre nos états mentaux et le monde extérieur, d'autre part. Par conséquent, rendre compte de la relation entre les raisons et la rationalité exigerait en quelque sorte de répondre à la question de savoir comment sont liés ces deux aspects de la normativité, et donc, en dernière instance (du moins en partie), à la question de savoir en quoi

(tel qu'il le formule) ne l'oblige pas à conclure ce qu'il conclut. N'admet-il pas lui-même que la possibilité pour un agent de reconnaître une implication immédiate et une inconsistance immédiate serait liée au fait de posséder une certaine disposition par rapport à des propositions? Il affirme: "I am inclined to take as fundamental certain dispositions to treat propositions in certain ways, in particular, dispositions to treat some propositions as immediately implying others and some as immediately inconsistent with each other" (Harman, 1986, p. 18). Selon Harman, quelqu'un a une raison de croire $P$ s'il reconnaît que $P$ est impliqué par ce qu'il croit. Supposons qu'un agent croie que $X$, et croie que si $X$ alors $P$, et qu'il reconnaisse l'implication. Du point de vue de l'agent qui raisonne (perspective de la $1^{\text {re }}$ personne), reconnaître l'implication ne consiste pas à reconnaître que $P$ est impliqué par le fait qu'il croit que $X$, et qu'il croit que si $X$ alors $P$, mais plutôt par le fait que $X$, et que si $X$ alors $P$. 
consiste raisonner. Paradoxalement, nous ne semblons pas disposer d'une compréhension claire de ce que fait l'agent qui raisonne ni, par conséquent, de la relation entre son raisonnement proprement dit et ses "raisons" de croire/d'agir, ou même de la relation entre son raisonnement et la rationalité de ses attitudes, ou, a fortiori, de la relation entre ses raisons de croire/d'agir et la rationalité de ses attitudes.

Comme nous l'avons vu, dire que les prémisses de l'argument ReCEM pouvant être associées au raisonnement proprement dit d'un agent correspondent aux raisons qu'il a de croire ce qu'il croit n'est pas équivalent à dire que ces raisons sont celles pour lesquelles ce qu'il croit est vrai (ou non). L'exemple d'Anna a permis de montrer que les prémisses de l'argument ReCEM pouvant être associées à son raisonnement proprement dit correspondent aux raisons qu'elle a de croire ce qu'elle croit, et non aux raisons pour lesquelles Sam n'a pas publié de livre chez Oxford. Anna se trompe, mais son raisonnement semble sensé: elle semble respecter les normes de rationalité. Toutefois, elle ne croit pas ce qu'elle devrait croire: c'est précisément là que se situe l'asymétrie normative. La difficulté posée par l'approche d'Harman est en quelque sorte de ne pas prendre en considération ce qui est impliqué par cette asymétrie normative et de s'en tenir principalement à déterminer les normes de rationalité qui gouvernent ou doivent gouverner les relations entre les états mentaux. Harman cherche principalement à déterminer les principes de révision que nous suivons ou devons suivre lorsque nous raisonnons correctement et montre, à juste titre, que ces principes ne sauraient être confondus avec ceux de la logique. Sa théorie du raisonnement a grandement contribué à ne plus concevoir le raisonnement en termes logiques et à insister sur son caractère psychologique, sur le fait qu'il s'agit d'une activité faite par des agents psychologiquement limités. Ce qui est juste. Mais il est temps maintenant de prendre acte du fait que les sujets psychologiques ont, précisément, une perspective sur le monde. La difficulté de son approche réside, par conséquent, dans le fait de négliger ce deuxième aspect de la normativité qui concerne cette fois les normes qui gouvernent les relations entre nos états mentaux et le monde extérieur. Cela concerne cet autre aspect du raisonnement qui, lui, est inférentiel. Inversement, la perspective de la $1^{\text {re }}$ personne permettrait de mettre en lumière cet autre aspect du raisonnement, et de montrer que, ce que l'agent met en relation lorsqu'il raisonne, ce sont les contenus propositionnels de certains de ses états mentaux: il met en relation des propositions dont la vérité est indépendante du fait qu'elles soient crues ou non par lui, ce qui concerne (cette fois) les normes qui règlent les relations entre ses états mentaux et le monde extérieur. C'est précisément ce qui permet d'expliquer pourquoi Anna se trompe, même si son raisonnement est sensé. Son raisonnement possède un double aspect, et c'est à ce double aspect qu'est liée l'asymétrie normative. Une théorie adéquate du raisonnement ne peut donc faire 
l'économie ni du point de vue de la $1^{\text {re }}$ personne ni de celui de la $3^{\mathrm{e}}$ personne. En cela réside tout le défi ${ }^{32}$.

\section{Bibliographie}

Anscombe, G.E.M. Intention, Oxford, Basil Blackwell, 1957.

Broome, John. "The Unity of Reasoning?", in S. Robertson (dir.), Spheres of Reason, Oxford, Oxford University Press, 2009, p. 62-91.

Brunet, Josée. "Le double aspect du raisonnement pratique ", Revue philosophique de Louvain, 101, 2003, p. 479-500.

Brunet, Josée. «La conception du raisonnement de John Broome: "Que nous exprimons-nous lorsque nous raisonnons?», Dialogue, XLVII, 2008, p. 633-662

Harman, Gilbert. «Practical Reasoning», Review of Metaphysics, 29, 1976, p. 431463.

-. Change in View, Cambridge (Mass.), MIT Press, 1986. «Rationality , in E.E. Smith et D.N. Oberson (dir.), Thinking: Invitation to Cognitive Science, vol. III, Cambridge (Mass.), MIT Press, 1995, p. 175-211. . Reasoning, Meaning and Mind, Oxford, Oxford University Press, 1999. . Notes on Practical Reasoning, 2007, Ms.

32. Dans la littérature plus récente, une des pistes à explorer est celle suggérée par John Broome, 2009, qui s'est intéressé à développer une théorie du raisonnement visant précisément à prendre en considération les points de vue de la $1^{\text {re }}$ et de la $3^{\text {e }}$ personne. Je discute de sa proposition dans Brunet, 2008. 